\title{
Increased serum neuregulin 4 levels in women with polycystic ovary syndrome: a case-control study
}

\author{
Muzaffer Temur ${ }^{1}$, Mehmet Calan ${ }^{2}$, Murat Akşit ${ }^{3}$, Özgür Yilmaz ${ }^{4}$, Tayfur Çift ${ }^{1}$, Burak Akselim ${ }^{1}$, \\ Burcu Dinçgez Çakmak ${ }^{1}$, Emin Üstünyurt ${ }^{1}$
}

\author{
${ }^{1}$ Bursa Yüksek Ihtisas Education and Research Hospital Department of Obstetrics and Gynecology, Bursa, Turkey \\ 2Izmir Bozyaka Education and Research Hospital, Department of Endocrinology, İzmir, Turkey \\ ${ }^{3}$ Tepecik Education and Research Hospital, Department of Medical Biochemistry, İzmir, Turkey \\ ${ }^{4}$ Manisa Merkezefendi State Hospital and Manisa Akhisar State Hospital, \\ Department of Obstetrics and Gynecology, Manisa, Turkey
}

\begin{abstract}
Objectives: Neuregulin 4 (NRG4) is an adipokine that is synthesized in many tissues and has been shown to be associated with the development of obesity and metabolic disorders in animals and humans. The aim of this study is to investigate the relationship between serum NRG4 levels and various metabolic parameters in women with PCOS.

Material and methods: This cross-sectional study included 40 women with PCOS and 40 age- and BMI-matched controls

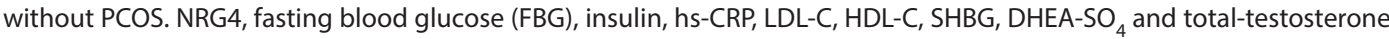
levels were measured in all the participants. HOMA-IR was used to calculate the insulin resistance.

Results: Serum NRG4 levels were higher in women with PCOS than in healthy women $(24.89 \pm 9.32$ [ng/mL] vs. $18.98 \pm 6.40$ [ng/mL], p = 0.002). FBG, LDL-C, HDL-C, LH, SHBG, FAI, DHEA-SO4, insulin, hs-CRP, HOMA-IR and total-testosterone levels were significantly higher in women with PCOS than controls. Circulating NRG4 levels were positively correlated with HOMA-IR, insulin and hs-CRP for both groups. There was a positive correlation between NRG4 and FBG in the PCOS group. HOMA-IR and hs-CRP were associated with NRG4.

Conclusion: The high concentration of circulating NRG4 in PCOS may be associated with insulin resistance and low-grade chronic inflammation.
\end{abstract}

Key words: Neuregulin 4, PCOS, hs-CRP, HOMA-IR

\section{INTRODUCTION}

Polycystic ovarian syndrome (PCOS) is a common endocrinopathy affecting 5-10 percent of women during their reproductive period and is characterized by ovulatory dysfunction, polycystic ovaries and biochemical (elevated androgens) and/or clinical (hirsutism, acne) hyperandrogenism. PCOS is a disease associated with insulin resistance, glucose intolerance, metabolic syndrome, obesity, hyperlipidemia, hypertension, chronic low-grade inflammation and increased risk of developing type 2 diabetes mellitus (T2DM) [1-4]. Although the etiopathogenesis of PCOS is not yet fully understood, insulin resistance and hyperinsulinemia play an important role in the etiopathogenesis of PCOS. In general, insulin resistance and hyperinsulinemia affect 70 percent of women with PCOS. Despite extensive research, the underlying mechanism of insulin resistance in PCOS has not been fully understood [5-8].

Neuregulin 4 (NRG4) is a member of the neuregulin protein family (NRG1-NRG4) and serves as an adipokine that is synthesized in many tissues, especially in brown adipose tissue (BAT). Similarly to other neuregulin members, NRG4 activates epidermal growth factor receptors (EGFR), also known as erbB receptors, and binds specifically to ErbB3 and ErbB4 receptors initiating cell-to-cell signaling via tyrosine phosphorylation $[9,10]$. NRG4 has been shown to have an effect in the development of obesity and metabolic disorders including T2DM and non-alcoholic fatty liver disease (NAFLD) in animals and 
humans [11, 12]. NRG4 reduces hepatic lipogenic signals and it maintains glucose and lipid homeostasis in obesity [12]. NRG4 levels in humans have been correlated with body fat and liver fat mass and have been associated with increased insulin sensitivity and reduced hepatic steatosis when on high-fat diet compared to controls [12]. Also, a decreased NRG4 expression in BAT may be associated with obesity, glucose intolerance and insulin resistance $[12,13]$. Yet, the role of NRG4 has not been fully understood in human homeostasis. Despite many studies on T2DM, metabolic syndrome and NRG4, the relationship between NRG4 and PCOS has not been researched yet. For this reason, the aim of this study is to investigate the relationship between serum NRG4 levels and various metabolic parameters in patients with PCOS.

\section{MATERIAL AND METHODS}

This cross-sectional clinical trial study was conducted in Bursa Yuksek Ihtisas Training and Research Hospital Gynecology and Obstetrics Clinic. The institutional ethical committee has approved the trial protocol. We enrolled in the study a total of 80 patients presenting to our gynecology outpatient clinics. The study group composed of 40 women diagnosed with PCOS (age and BMI matched) while the control group consisted of age and BMI matched 40 women that had regular menstrual cycles. Detailed history of all patients was taken. After a physical exam we recorded anthropometric data (age, weight, height and waist circumference). Height [cm] and weight [kg] were measured with the patient barefoot in light daily clothes. BMI was calculated using the formula: weight $[\mathrm{kg}] /$ square meter of height $\left[\mathrm{m}^{2}\right]$. At the end of a gentle expiration, the waist circumference $[\mathrm{cm}]$ was measured at the level halfway between the lower rib and the iliac crest. Blood pressure was measured in the sitting position ensuring a min. of 15 [min] rest prior to measurement. The average of three measurements taken was calculated. The PCOS diagnosis was confirmed with at least two criteria according to the 2003 Rotterdam consensus: oligo-ovulation or anovulation, presence of polycystic ovaries on transvaginal ultrasound and hyperandrogenism present as a laboratory finding and/or symptoms. According to a clinical Ferriman-Gallwey hyperandrogenism scoring system patients should have a minimum score of 8 and the biochemical hyperandrogenism was defined as total testosterone levels above the range that is regarded as normal (normal range: $0.52-2.42 \mathrm{nmol} / \mathrm{L})[14,15]$. For the evaluation of the polycystic ovaries'(PCO) morphology, all women underwent transvaginal ultrasonography in the early follicular phase. The presence of PCO was diagnosed with the presence of 12 or more follicles with a diameter of 2-9 $\mathrm{mm}$ and/or increased ovarian volume $\left(>10 \mathrm{~cm}^{3}\right)$ of each ovary. Menstrual periods were characterized by oligomenorrhea (absence of menstruation for 45 days or more) or by amenorrhea (no menstrual period for 3 months or more) in the PCOS group. The control group menstrual periods were regular and lasted 25-32 days. Additionally, the control group had neither hirsutism nor hyperandrogenism. All participants were non-smokers and did not have any medical treatment in the past. Pregnant or lactating women, hyperprolactinemia, Cushing's syndrome, congenital adrenal hyperplasia, other diseases of the adrenal gland, thyroid disorders, impaired glucose tolerance, type 1 or type 2 diabetes mellitus and the use of insulin sensitizers or oral anti-diabetics were all excluded from the study.

\section{Laboratory studies}

Antecubital venous blood samples were taken from each female in the early follicular phase of menstruation (3rd to 5 th day). The samples were centrifuged and separated from the serum and stored at $-80^{\circ} \mathrm{C}$ for analysis. High-density lipoprotein cholesterol (HDL-C), low-density lipoprotein cholesterol (LDL-C), total testosterone, triglycerides, high-density lipoprotein cholesterol (HDL-C), fasting blood glucose (FBG), serum insulin, Dehydroepiandrosterone sulfate (DHEA-S), follicle stimulating hormone (FSH), luteinizing hormone (LH), estradiol (E2) and NRG4 levels were measured. FBG, serum hs-CRP, total cholesterol, total HDL-C and triglyceride levels were determined using an automated analyzer (Abbott Architect C 16000, IL, USA) with its own kits (Abbott Diagnostics, Wiesbaden, Germany). LDL-C was calculated using the Friedewald equation: $\mathrm{LDL}-\mathrm{C}=$ total cholesterol- $(\mathrm{HDL}-\mathrm{C})$ - triglyceride/5. Non-HDL-C was calculated as HDL-C subtracted from total cholesterol. Serum insulin levels were measured by an automated analyzer (Abbott Architect I2000, IL, USA) using a chemiluminescent microparticle immunoassay (CMIA) with its own kit (Abbott Diagnostics, Wiesbaden, Germany). Serum FSH, LH, E2, total testosterone and DHEA-S levels were measured with CMIA (Beckman Coulter Inc., Brea, CA, USA). Serum NRG4 concentrations were assessed by enzyme immunoassay (Elabscience, Wuhan, Hubei, China Catalog Number: E-EL-H0890). The intra-assay CV was $<10 \%$ and the inter-assay $\mathrm{CV}$ was $<12 \%$. Using the formula stated for the homoeostasis model assessment of insulin resistance (HOMA-IR) method, the insulin resistance was calculated according to the formula: HOMA-IR = fasting insulin $[\mathrm{mU} / \mathrm{mL}] \times$ fasting glucose $[\mathrm{mg} / \mathrm{dL}] /$ /405 [16]. The free androgen index (FAl) was obtained as the quotient 100 testosterone/SHBG.

\section{Statistical analysis}

All statistical analysis was performed using the Statistical Package for the Social Sciences Software, version 18.0 (SPSS Inc., Chicago, IL. USA). The normality of the data was tested using the Kolmogorov-Smirnov test and all continuous variables had normal distribution ( $p>0.05$ ). Continuous variables were presented as mean \pm standard deviation (SD). The demographic and laboratory characteristics of women with and without PCOS were compared using the two-tailed independ- 
ent sample t-test. The relationship between NRG4 and other parameters was assessed by Pearson correlation analysis. To investigate the relationship between independent NRG4 levels in PCOS development, multiple linear regression analyzes were performed to adjust for equivalence and to determine the independent relationships of NRG4 levels with age, BMI, HOMA-IR, FAI and hs-CRP. All independent variables in the multiple linear regressions were tested for multicollinearity. If the variance inflation factor (VIF) exceeded 2.5, the variable was considered to be collinear. All reported confidence interval $(\mathrm{Cl})$ values are calculated at the $95 \%$ level. A two-sided $p$ value of $<0.05$ was considered statistically significant.

\section{RESULTS}

\section{Clinical and laboratory characteristics of the study population}

The comparative demographic and laboratory parameters of women with and without PCOS are presented in
Table 1. The cases were matched in terms of age and BMI. Both parameters were similar and no statically significant differences were recorded between the groups ( $p>0.05)$.

Serum NRG4 levels were higher in women with PCOS than in healthy women and the difference was statically significant $(24.89 \pm 9.32 \mathrm{ng} / \mathrm{mL}$ vs. $5.29 \pm 1.45 \mathrm{ng} / \mathrm{mL}$, $\mathrm{p}=0.002$ ). Serum FBG, LDL-C, HDL-C Triglyceride, LH, SHBG, FAI, DHEA-SO4, insulin, hs-CRP, HOMA-IR, total testosterone levels and Ferriman-Gallwey score were all significantly higher in women with PCOS than in healthy women.

\section{Correlation analysis}

Correlation was calculated between NRG4 and various other parameters in women with PCOS. Pearson's correlation coefficients are presented in Table 2. Circulating NRG4 levels were positively correlated with HOMA-IR, insulin and hs-CRP in both groups. There was a positive correlation between NRG4 and FBG in the PCOS group. Table 1. Comparison of demographic and laboratory characteristics of patients

\begin{tabular}{|c|c|c|c|}
\hline Variables & $\begin{array}{l}P C O S \\
n=40\end{array}$ & $\begin{array}{c}\text { Controls } \\
n=40\end{array}$ & $\mathbf{p a}^{\mathbf{a}}$ \\
\hline Age (years) & $27.54 \pm 4.62$ & $26.75 \pm 4.73$ & 0.453 \\
\hline BMI $\left[\mathrm{kg} / \mathrm{m}^{2}\right]$ & $27.85 \pm 4.37$ & $27.57 \pm 4.30$ & 0.775 \\
\hline Waist circumference $[\mathrm{cm}]$ & $88.20 \pm 10.43$ & $87.82 \pm 10.01$ & 0.546 \\
\hline $\mathrm{SBP}[\mathrm{mm} \mathrm{Hg}]$ & $108.70 \pm 12.63$ & $107.93 \pm 11.90$ & 0.780 \\
\hline $\mathrm{DBP}[\mathrm{mm} \mathrm{Hg}]$ & $74.82 \pm 6.64$ & $73.80 \pm 5.77$ & 0.465 \\
\hline Ferriman-Gallwey score & $14.36 \pm 3.25$ & $4.13 \pm 1.24$ & $<0.001^{*}$ \\
\hline $\mathrm{FBG}[\mathrm{mg} / \mathrm{dL}]$ & $87.97 \pm 6.69$ & $82.72 \pm 6.47$ & $0.001^{*}$ \\
\hline Insulin $[\mu \mid \mathrm{U} / \mathrm{mL}]$ & $20.33 \pm 6.45$ & $11.77 \pm 4.26$ & $<0.001^{*}$ \\
\hline HOMA-IR & $4.40 \pm 1.41$ & $2.38 \pm 0.83$ & $<0.001^{*}$ \\
\hline Total cholesterol [mg/dL] & $221.90 \pm 28.55$ & $208.58 \pm 41.33$ & 0.098 \\
\hline LDL-C [mg/dL] & $150.16 \pm 24.53$ & $135.00 \pm 26.58$ & $0.010^{*}$ \\
\hline $\mathrm{HDL}-\mathrm{C}[\mathrm{mg} / \mathrm{dL}]$ & $41.20 \pm 8.57$ & $50.65 \pm 9.95$ & $<0.001^{*}$ \\
\hline Triglycerides [mg/dL] & $152.68 \pm 31.17$ & $114.66 \pm 27.45$ & $<0.001^{*}$ \\
\hline hs-CRP [mg/L] & $1.41 \pm 0.49$ & $0.70 \pm 0.18$ & $<0.001^{*}$ \\
\hline $\mathrm{FSH}[\mathrm{mIU} / \mathrm{mL}]$ & $7.43 \pm 1.63$ & $7.47 \pm 1.88$ & 0.924 \\
\hline $\mathrm{LH}[\mathrm{mlU} / \mathrm{mL}]$ & $12.88 \pm 3.40$ & $7.82 \pm 2.68$ & $<0.001^{*}$ \\
\hline Estradiol [pg/mL] & $54.56 \pm 11.37$ & $50.35 \pm 7.33$ & 0.053 \\
\hline Total-testosterone [nmol/L] & $3.04 \pm 0.39$ & $1.74 \pm 0.34$ & $<0.001^{*}$ \\
\hline SHBG [nmol/L] & $41.03 \pm 11.29$ & $70.46 \pm 13.86$ & $<0.001^{*}$ \\
\hline FAI (\%) & $7.80 \pm 1.75$ & $2.47 \pm 0.09$ & $<0.001^{*}$ \\
\hline DHEA-SO ${ }_{4}[\mu \mathrm{g} / \mathrm{dL}]$ & $195.55 \pm 56.73$ & $158.94 \pm 36.47$ & $0.001^{*}$ \\
\hline Neuregulin-4 [ng / mL] & $24.89 \pm 9.32$ & $18.98 \pm 6.40$ & $0.002^{*}$ \\
\hline \multicolumn{4}{|c|}{$\begin{array}{l}\text { Results are given in mean } \pm \text { SD. }{ }^{a} \text { Independent samples t-test was used. A } P \text { value of }<0.05 \text { was considered significant*. BMI }- \text { body mass index; DHEA-S } \\
\text { - dehydroepiandrosterone sulfate; DBP - diastolic blood pressure; FAI - free androgen index; FBG - fasting blood glucose; FSH - follicle-stimulating hormone; } \\
\mathrm{HDL}-\mathrm{C}-\text { high density lipoprotein cholesterol; HOMA-IR - homeostasis model assessment of insulin resistance; hs-CRP - high sensitivity C-reactive protein; LDL-C } \\
\text { - low density lipoprotein cholesterol; LH - luteinizing hormone; PCOS - polycystic ovary syndrome; SBP - systolic blood pressure; SHBG - sex hormone-binding } \\
\text { globulin }\end{array}$} \\
\hline
\end{tabular}


Table 2. Correlation coefficients between neuregulin-4 levels and clinical parameters

\begin{tabular}{|l|c|c|c|c|}
\hline \multirow{2}{*}{} & \multicolumn{4}{|c|}{ Neuregulin-4 } \\
\cline { 2 - 5 } & \multicolumn{2}{|c}{ PCOS } & \multicolumn{2}{c|}{ Control } \\
\hline Age & 0.156 & 0.327 & 0.243 & 0.264 \\
\hline BMI & 0.163 & 0.478 & 0.257 & 0.268 \\
\hline Waist circumference & 0.257 & 0.312 & 0.241 & 0.198 \\
\hline SBP & 0.113 & 0.235 & 0.101 & 0.356 \\
\hline DBP & 0.254 & 0.167 & 0.178 & 0.215 \\
\hline Insulin & 0.298 & $0.017^{*}$ & 0.201 & $0.029^{*}$ \\
\hline FBG & 0.135 & $0.046^{*}$ & 0.112 & 0.052 \\
\hline HOMA-IR & 0.243 & $0.035^{*}$ & 0.125 & $0.041^{*}$ \\
\hline FAl & 0.143 & 0.231 & 0.216 & 0.228 \\
\hline hs-CRP & 0.326 & $0.007^{*}$ & 0.114 & $0.038^{*}$ \\
\hline Total cholesterol & 0.264 & 0.167 & 0.124 & 0.127 \\
\hline LDL-C & 0.312 & 0.169 & 0.278 & $0.431^{*}$ \\
\hline HDL-C & 0.218 & 0.128 & 0.376 & 0.127 \\
\hline Triglycerides & 0.271 & 0.472 & 0.367 & 0.145 \\
\hline Persing & &
\end{tabular}

Pearson's correlation analysis was used. $r$ - Pearson's correlation coefficient; A P value of $<0.05$ was considered significant $\left({ }^{*}\right)$. BMI — body mass index; DBP — diastolic blood pressure; FAI — free androgen index; FBG - fasting blood glucose; HDL-C — high-density lipoprotein cholesterol; HOMA-IR - homeostasis model assessment of insulin resistance: hs-CRP - high sensitivity C-reactive protein; LDL-C — low-density lipoprotein cholesterol; SBP — systolic blood pressure

\section{Multivariate regression analysis}

A linear regression model was established using independent variables that can influence the levels of NRG4, such as age, hs-CRP, HOMA-IR, BMI and FAl. According to this model HOMA-IR and Hs-CRP were associated with NRG4 levels (Tab. 3).

\section{DISCUSSION}

In this present study we have demonstrated for the first time that circulating NRG4 levels were significantly higher in women with PCOS when compared to control patients. In patients with PCOS NRG4 was positively correlated with insulin resistance marker HOMA-IR, inflammatory marker hs-CRP, insulin and with FBG. In addition, multivariate regression analysis has shown that HOMA-IR and hs-CRP were both independent factors influencing serum NRG4 levels.

NRG4 is an adipokine that is similar to other neuregulin family members. It activates epidermal growth factor receptor (EGFR), also known as erbB receptor, and is synthesized in many tissues, especially in BAT $[12,17]$. The hypothalamic-pituitary-gonadal axis and the luteinizing hormone-releasing hormone $(\mathrm{LHRH})$ are required for healthy pubertal development [18, 19]. ErbB receptors have been shown to play an important role in the healthy functioning of LHRH. In mice with neuregulin-ErbB4/2 receptor deficiency the required LHRH failed to be secreted so the sex development of mice was impaired [20]. Day et al. reported in their genetic study that ErbB2, ErbB3 and ErbB4 receptors may be associated with PCOS [21]. In another animal study, overexpressed NRG4 has been shown to cause an increase in insulin sensitivity while NRG4 deficiency has been shown to cause insulin resistance. Also, a different study on animals reports that NRG4 causes insulin secretion in Beta islet cells $[12,22]$. Ma et al. report that NRG4 expressed in NRG4-transgenic mice was protective against insulin resistance and obesity [23]. Although NRG4 is a novel adipokine and studies on humans have been limited, Cai et al. showed that circulating NRG4 levels in patients with metabolic syndrome were found to be lower than in control group. An inverse correlation was found between the level of NRG4 and the risk of metabolic syndrome with insulin resistance [24]. Jiang et al. reported that Serum NRG4 levels were associated with subclinical atherosclerosis in obese adults [25]. Kang et al. have found that there was no significant difference in the serum NRG4 levels when comparing type 2 diabetic patients with non-diabetic patients. Yet, there was a positive correlation between NRG4 levels and FBG, HOMA-IR and triglyceride levels [17]. In contrast, Zhang et al. have reported that type 2 diabetic patients were found to have

Table 3. Evaluation of the association of Neuregulin-4 with age, BMI, HOMA-IR, FAl and Hs-CRP in the study population (women with and without PCOS) using multiple linear regression analysis

\begin{tabular}{|c|c|c|c|c|}
\hline \multirow[b]{2}{*}{ Variables } & \multirow[t]{2}{*}{$\beta$} & \multicolumn{2}{|c|}{$95 \% \mathrm{Cl}$} & \multirow[t]{2}{*}{$\mathbf{P}$} \\
\hline & & Lower & Upper & \\
\hline Age & 0.685 & -1.786 & 3.156 & 0.564 \\
\hline BMI & 0.877 & -0.781 & 2.542 & 0.327 \\
\hline HOMA-IR & 3.234 & 1.684 & 4.785 & $0.014^{*}$ \\
\hline FAI & 2.656 & -2.584 & 7.896 & 0.276 \\
\hline hs-CRP & 3.614 & 1.304 & 5.925 & $0.008^{*}$ \\
\hline
\end{tabular}

Multiple linear regression analysis was used. $\beta$ - Unstandardized regression coefficient; $\mathrm{Cl}$ - confidence interval. A $P$ value of $<0.05$ was considered significant $(*)$. $\mathrm{BMI}$ — body mass index; FAI — free androgen index; DBP — diastolic blood pressure; HOMA-IR — homeostasis model assessment of insulin resistance; hs-CRP — high sensitivity C-reactive protein 
a higher NRG4 level than the control group. Moreover, there was an inverse correlation between NRG4 levels and FBG, insulin and HOMA-IR [13]. Many studies in the literature show that NRG 4 indirectly affects the hormonal environment by binding to erbB receptors. In our study NRG4 level was significantly higher in patients with PCOS compared to control group. In addition, there was a positive correlation of circulating NRG4 level with HOMA-IR, insulin, FBG and hs-CRP. According to the multivariate regression analysis of our study, HOMA-IR and hs-CRP were associated with NRG4. We may hypothesize that there could be a problem in the interaction of NRG4 with erbB receptors in patients with PCOS and thus the high concentration of circulating NRG4 levels, which may be associated with insulin resistance in PCOS.

PCOS is a low-grade chronic inflammatory disease. Many studies have shown a relationship between PCOS and inflammation markers $[7,8,26]$. There is also a relationship between NRG4 and chronic inflammatory state. Studies on animals with ulcerative colitis and Crohn's disease have shown that NRG4 treated animals showed a decreased inflammation in the intestines and a decreased apoptosis in intestinal cells [27, 28]. Ma et al. report that NRG4-transgenic mice were found to have a decreased expression of monocyte chemotactic protein 1 (Mcp1) and a decreased expression of macrophage-specific genes [19]. Similarly, our study has found that PCOS patients had higher levels of hs-CRP than controls. There was also a positive correlation between hs-CRP and NRG4 levels. Moreover, there was a significant relationship between NRG4 and hs-CRP levels. Consequently, it may be that in patients with PCOS the NRG4 levels are increasing as compensation mechanism to low-grade chronic inflammation during this disease.

In conclusion, NRG4 levels were found to be significantly higher in women with PCOS. There was also a significant correlation between HOMA-IR, hs-CRP and NRG4. Elevation of NRG4 levels in PCOS may be associated with increased insulin resistance and low-grade chronic inflammation. Our study is the first study in the literature to investigate such relationship. More detailed studies are needed to understand the association and the role of NRG4 in PCOS.

\section{Disclosure statement}

The authors declare that there are no conflicts of interest.

\section{REFERENCES}

1. Legro RS, Arslanian SA, Ehrmann DA, et al. Endocrine Society. Diagnosis and treatment of polycystic ovary syndrome: an Endocrine Society clinical practice guideline. J Clin Endocrinol Metab. 2013; 98(12): 4565-4592, doi: 10.1210/jc.2013-2350, indexed in Pubmed: 24151290.

2. Jayasena $\mathrm{CN}$, Franks $\mathrm{S}$. The management of patients with polycystic ovary syndrome. Nat Rev Endocrinol. 2014; 10(10): 624-636, doi: 10.1038/nrendo.2014.102, indexed in Pubmed: 25022814.
3. Ehrmann DA. Polycystic ovary syndrome. N Engl J Med. 2005; 352(12): 1223-1236, doi: 10.1056/NEJMra041536, indexed in Pubmed: 15788499.

4. Azziz R, Woods KS, Reyna R, et al. The prevalence and features of the polycystic ovary syndrome in an unselected population. J Clin Endocrinol Metab. 2004; 89(6): 2745-2749, doi: 10.1210/jc.2003-032046, indexed in Pubmed: 15181052.

5. Goodarzi MO, Dumesic DA, Chazenbalk G, et al. Polycystic ovary syndrome: etiology, pathogenesis and diagnosis. Nat Rev Endocrinol. 2011;7(4): 219-231, doi: 10.1038/nrendo.2010.217, indexed in Pubmed: 21263450.

6. Mani H, Levy MJ, Davies MJ, et al. Diabetes and cardiovascular events in women with polycystic ovary syndrome: a 20-year retrospective cohort study. Clin Endocrinol (Oxf). 2013; 78(6): 926-934, doi: 10.1111/cen.12068, indexed in Pubmed: 23046078.

7. Tarkun I, Arslan BC, Cantürk Z, et al. Endothelial dysfunction in young women with polycystic ovary syndrome: relationship with insulin resistance and low-grade chronic inflammation. J Clin Endocrinol Metab. 2004; 89(11): 5592-5596, doi: 10.1210/jc.2004-0751, indexed in Pubmed: 15531516.

8. Demir B, Cengiz H, Ungan I, et al. The relationship between epicardial adipose tissue thickness and oxidative stress parameters in patients with isolated polycystic ovary syndrome. Gynecol Endocrinol. 2015; 31(7): 531-535, doi: 10.3109/09513590.2015.1018160, indexed in Pubmed: 26340353.

9. Harari D, Tzahar E, Romano J, et al. Neuregulin-4: a novel growth factor that acts through the ErbB-4 receptor tyrosine kinase. Oncogene. 1999; 18(17): 2681-2689, doi: 10.1038/sj.onc.1202631, indexed in Pubmed: 10348342.

10. Britsch $\mathrm{S}$. The neuregulin-1/ErbB signaling system in development and disease. Adv Anat Embryol Cell Biol. 2007; 190: 1-65, doi: 10.1007/9783-540-37107-6, indexed in Pubmed: 17432114.

11. Wang GX, Zhao XY, Lin JD. The brown fat secretome: metabolic functions beyond thermogenesis. Trends Endocrinol Metab. 2015; 26(5): 231-237, doi: 10.1016/j.tem.2015.03.002, indexed in Pubmed: 25843910.

12. Wang GX, Zhao XY, Meng ZX, et al. The brown fat-enriched secreted factor Nrg4 preserves metabolic homeostasis through attenuation of hepatic lipogenesis. Nat Med. 2014; 20(12): 1436-1443, doi: 10.1038/nm.3713, indexed in Pubmed: 25401691.

13. Zhang $L, F u ~ Y, Z$ hou N, et al. Circulating neuregulin 4 concentrations in patients with newly diagnosed type 2 diabetes: a cross-sectional study. Endocrine. 2017 [Epub ahead of print]; 57(3): 535-538, doi: 10.1007/s12020-017-1324-3, indexed in Pubmed: 28523627.

14. Rotterdam ESHRE/ASRM-Sponsored PCOS Consensus Workshop Group. Revised 2003 consensus on diagnostic criteria and long-term health risks related to polycystic ovary syndrome. Fertil Steril. 2004; 81: 19-25.

15. FERRIMAN D, GALLWEY JD. Clinical assessment of body hair growth in women. J Clin Endocrinol Metab. 1961; 21: 1440-1447, doi: 10.1210/jcem-21-11-1440, indexed in Pubmed: 13892577.

16. Matthews DR, Hosker JP, Rudenski AS, et al. Homeostasis model assessment: insulin resistance and beta-cell function from fasting plasma glucose and insulin concentrations in man. Diabetologia. 1985; 28(7): 412-419, doi: 10.1007/bf00280883, indexed in Pubmed: 3899825.

17. Kang YE, Kim JiM, Choung S, et al. Comparison of serum Neuregulin 4 (Nrg4) levels in adults with newly diagnosed type 2 diabetes mellitus and controls without diabetes. Diabetes Res Clin Pract. 2016; 117: 1-3, doi: 10.1016/j.diabres.2016.04.007, indexed in Pubmed: 27329015.

18. Ojeda S, Urbanski H, Katz K, et al. Activation of Estradiol-Positive Feedback at Puberty: Estradiol Sensitizes the LHRH-Releasing System at Two Different Biochemical Steps. Neuroendocrinol. 1986; 43(2): 259-265, doi: 10.1159/000124535.

19. Ojeda SR, Urbanski HF, Costa ME, et al. Involvement of transforming growth factor alpha in the release of luteinizing hormone-releasing hormone from the developing female hypothalamus. Proc Natl Acad Sci U S A. 1990; 87(24): 9698-9702, doi: 10.1073/pnas.87.24.9698, indexed in Pubmed: 2263621.

20. Prevot $\mathrm{V}$, Rio $\mathrm{C}, \mathrm{Cho} \mathrm{GJ}$, et al. Normal female sexual development requires neuregulin-erbB receptor signaling in hypothalamic astrocytes. J Neurosci. 2003; 23(1): 230-239, indexed in Pubmed: 12514220.

21. Day FR, Hinds DA, Tung JY, et al. Causal mechanisms and balancing selection inferred from genetic associations with polycystic ovary syndrome. Nat Commun. 2015; 6: 8464, doi: 10.1038/ncomms9464, indexed in Pubmed: 26416764.

22. South JCM, Blackburn E, Brown IR, et al. The neuregulin system of ligands and their receptors in rat islets of langerhans. Endocrinology. 2013; 
154(7): 2385-2392, doi: 10.1210/en.2012-2133, indexed in Pubmed: 23610133.

23. Ma Y, Gao M, Liu D. Preventing High Fat Diet-induced Obesity and Improving Insulin Sensitivity through Neuregulin 4 Gene Transfer. Sci Rep. 2016; 6: 26242, doi: 10.1038/srep26242, indexed in Pubmed: 27184920.

24. Cai $C$, Lin $M, X u Y$, et al. Association of circulating neuregulin 4 with metabolic syndrome in obese adults: a cross-sectional study. BMC Med. 2016; 14(1): 165, doi: 10.1186/s12916-016-0703-6, indexed in Pubmed: 27772531.

25. Jiang J, Lin $M, X u Y$, et al. Circulating neuregulin 4 levels are inversely associated with subclinical cardiovascular disease in obese adults. Sci Rep. 2016; 6: 36710, doi: 10.1038/srep36710, indexed in Pubmed: 27819316.
26. Dehdashtihaghighat S, Mehdizadehkashi A, Arbabi A, et al. Assessment of C-reactive Protein and C3 as Inflammatory Markers of Insulin Resistance in Women with Polycystic Ovary Syndrome: A Case-Control Study. J Reprod Infertil. 2013; 14(4): 197-201, indexed in Pubmed: 24551574.

27. McElroy SJ, Castle SL, Bernard JK, et al. The ErbB4 ligand neuregulin-4 protects against experimental necrotizing enterocolitis. Am J Pathol 2014; 184(10): 2768-2778, doi: 10.1016/j.ajpath.2014.06.015, indexed in Pubmed: 25216938.

28. Bernard JK, McCann SP, Bhardwaj V, et al. Neuregulin-4 is a survival factor for colon epithelial cells both in culture and in vivo. J Biol Chem. 2012; 287(47): 39850-39858, doi: 10.1074/jbc.M112.400846, indexed in Pubmed: 23033483. 